\title{
Pandémie et communautés minoritaires marginalisées : vers une approche inclusive en santé publique?
}

\author{
Cécile Rousseau $^{1}$ (D) Annie Jaimes ${ }^{2,3} \cdot$ Salam El-Majzoub ${ }^{4}$ \\ Received: 25 June 2020 / Accepted: 17 September 2020 / Published online: 6 October 2020 \\ (C) The Canadian Public Health Association 2020
}

\section{Résumé}

La pandémie a mis en lumière la complexité des mesures de santé publique et de leurs effets collatéraux, l'intrication des phénomènes sanitaires, sociaux et politiques sous-jacents, ainsi que leur spécificité pour des communautés minoritaires marginalisées. Fort de cette expérience, le présent commentaire souligne la pertinence d'élaborer des mécanismes de consultation qui tiennent compte de l'ensemble de la population en incluant les groupes minoritaires marginalisés, pour mieux informer les processus décisionnels en temps de pandémie. Dans un contexte de crise fluide, tout processus de consultation doit tenir compte des limites du possible et faire le deuil de l'exhaustivité au profit d'itérations répétées de dialogue, de médiation et de prises de décision au sujet de l'action. Il s'agit de créer des conditions propices à ce que les voix des personnes et groupes plus vulnérables soient entendus par les autorités publiques. Afin de nous préparer aux prochaines crises, il est nécessaire d'ores et déjà de développer des liens forts entre institutions publiques et communautés, tant majoritaires que minoritaires, afin de définir les mécanismes favorisant l'émergence d'une santé publique réellement inclusive, qui tienne compte de la santé physique, mentale et sociale de la population.

\begin{abstract}
The pandemic has highlighted the complexity of public health measures and their side effects, the intricacy of the underlying health, social and political phenomena at play as well as their specificity for marginalized minority communities. Based on this experience, this commentary suggests that it would be relevant in the future to develop consultation mechanisms that take into account the entire population, including marginalized minority groups, in order to better inform decision-making processes in times of pandemic. In a context of fluid crisis, any consultation process must take into account the limits of what is possible and mourn the comprehensiveness in favour of repeated iterations of dialogue, mediation and decision-making. This means creating conditions favourable to the consideration of the most vulnerable people and groups' voices by public authorities. In order to prepare for the next crises, it is necessary to develop strong links between public institutions and communities, both of majorities and minorities, in order to define the mechanisms favouring the emergence of truly inclusive public health, taking into account the physical, mental and social health of the population.
\end{abstract}

Mots-clés Pandémie $\cdot$ COVID-19 $\cdot$ sécurité publique $\cdot$ communautés $\cdot$ minorités

Keywords Pandemic $\cdot$ COVID-19 $\cdot$ Public safety $\cdot$ Communities $\cdot$ Minorities

Cécile Rousseau

cecile.rousseau@mcgill.ca

Annie Jaimes

Annie.jaimes@mail.mcgill.ca

Salam El-Majzoub

Salam.el-majzoub@mail.mcgill.ca
1 Division de psychiatrie sociale et culturelle, Université McGill, CLSC Parc-Extension, 7085 Hutchison, Bureau 204.2, Montréal, QC H3N 1Y9, Canada

2 Center for Refugee Studies, York University, Toronto, Canada

3 Sherpa University Institute, Montreal, Canada

4 Résidente en psychiatrie (R3), McGill University, Montreal, Canada 
Durant la première phase de la pandémie de la COVID-19, l'urgence de limiter la transmission du virus a fait l'objet d'un consensus social et politique dans de nombreux pays, dont au Canada. Le Québec n'a pas fait exception, et la population a bien adhéré aux mesures de confinement mises en place par la Direction de la santé publique. La pandémie a toutefois mis en lumière la complexité des effets collatéraux de ces mesures, ainsi que l'intrication des phénomènes sanitaires, sociaux et politiques sous-jacents. Alors que les impacts du confinement sont mieux connus, qu'on prépare la rentrée scolaire et qu'on jongle avec le déconfinement, tout en guettant les signes d'une deuxième vague, il devient nécessaire de réfléchir aux principes qui devraient sous-tendre les processus décisionnels en temps de pandémie. Le présent commentaire souligne l'importance pour le Québec d'élaborer des mécanismes de consultation qui tiennent compte de l'ensemble de la population et plus particulièrement des groupes minoritaires marginalisés. La crise de la COVID-19 se présente ainsi comme l'occasion de penser une approche plus inclusive et une meilleure préparation à d'autres crises du même ordre.

\section{Impacts de la pandémie sur les communautés minoritaires marginalisés}

En comparaison avec la population générale, les personnes appartenant à des groupes minoritaires ont vu leur santé physique plus affectée par la pandémie (morbidité et mortalité) des écarts notamment attribuables aux facteurs socio-économiques et au niveau d'exposition au virus à cause de leurs occupations et conditions de vie (Azar et al. 2020). À Montréal, les données disponibles suggèrent que les personnes issues de minorités linguistiques et culturelles, ainsi que les personnes vulnérables sur les plans socio-économique ou politique, rencontrent des obstacles structurels importants face à la crise (Cleveland et al. 2020). En effet, les conséquences du confinement et les problèmes économiques associés ont été plus marqués chez certains groupes minoritaires, tels les allophones et les migrants à statut précaire. Ces groupes, cumulant plusieurs facteurs de risques non-médicaux (socio-économiques, professionnels, linguistiques ou migratoire), ont rencontré des obstacles dans l'accès à l'information et dans la compréhension des consignes de santé publiques, souvent peu compatibles avec leurs conditions de (sur)vie (Institut national d'excellence en santé et en services sociaux 2020). À titre d'exemple, des personnes en demande d'asile travaillant en CHSLD (centres d'hébergement de soins de longue durée) se sont trouvées démunies devant le manque de matériel de protection ou d'information, une situation parfois aggravée par les barrières linguistiques et l'absence d'alternative advenant une perte d'emploi. Ces difficultés ont mis en évidence qu'une action de santé publique forte et cohérente devait considérer tous les membres de la population - tout particulièrement les communautés plus vulnérables face aux enjeux sanitaires, économiques ou socio-politiques sous-jacents à la crise.

\section{Impacts de la pandémie sur les relations intra- et intercommunautaires}

Essentiels face à l'adversité, les liens sociaux se trouvent fragilisés en contexte de pandémie par la menace de transmission et par le confinement, et ce plus sévèrement chez les groupes minoritaires vulnérables (Cohn and Kutalek 2016). $\mathrm{Au}$ Québec, des observations préliminaires indiquent en effet que la pandémie a ébranlé les réseaux d'entraide de diverses communautés (réseaux familiaux, amicaux, professionnels, scolaires, parascolaires, communautaires et religieux). L'interdiction de se regrouper a touché plusieurs communautés, notamment celles pour lesquelles la spiritualité joue un grand rôle, et qui se sont vues interdire de facto les pratiques spirituelles collectives - célébrations, rites funéraires, etc. (Gunnell et al. 2020; World Health Organization 2020). Face à ces défis, les organisations communautaires et religieuses se sont rapidement réorganisées et ont développé des stratégies alternatives permettant de maintenir les liens entre membres (ex : groupes et célébrations religieuses en ligne (Campbell 2020). Par ailleurs, plusieurs stratégies visant à préserver le bien-être des personnes ont été mises en place dans le cadre d'initiatives citoyennes. Par exemple, les communautés chinoise et iranienne ont mis en place un réseau pour accueillir les voyageurs en quarantaine, faire leur épicerie, etc. Ces stratégies, bien que prometteuses, ne sont pas une panacée, surtout dans le cas des réseaux virtuels, dans la mesure où leur impact est limité par des facteurs culturels (familiarité et confort) et structurels (littéracie informatique, connexion internet, ordinateur/portable).

Sur le plan intercommunautaire, la pandémie de la COVID-19 a également attisé des tensions. Les études sur les épidémies du sida, d'Ebola, du H1N1 ont bien documenté les phénomènes sociaux menant à une quête de boucs émissaires, à des dynamiques de blâme et théories du complot, ciblant le plus souvent des groupes minoritaires vulnérables (Atlani-Duault et al. 2015; Roy et al. 2020). Depuis le début de la pandémie actuelle, la commission des droits de la personne et des droits de la jeunesse du Québec (2020) a d'ailleurs documenté une augmentation des crimes haineux et gestes de discrimination visant des personnes de minorité (asiatique, juives hassidiques), en plus des travailleurs de la santé et personnes âgées. Il importe de penser les diverses tensions intercommunautaires qui peuvent fragiliser le tissu social d'une collectivité, aggraver les crises sanitaires, et contribuer à fragiliser les personnes et communautés plus vulnérables. 


\section{La voix des minorités dans l'établissement des priorités lors du confinement et du déconfinement}

Diverses solutions ont été proposées pour mitiger les conséquences du confinement sur la santé mentale de la population du Québec. Les solutions proposées visent surtout à promouvoir de saines habitudes de vie et à recourir aux services disponibles dans le réseau de la santé et des services sociaux, en privilégiant des approches virtuelles autant que faire se peut (Institut national d'excellence en santé et en services sociaux 2020; Pfefferbaum and North 2020). Globalement, les mesures de déconfinement à géométrie variable ont visé un équilibre coût/bénéfice. Mais comment choisir s'il faut d'abord ouvrir, après les petits commerces, les salles de gym, les centres communautaires, les églises et temples religieux? Les facteurs considérés déterminants pour le bien-être des personnes et des communautés, les priorités publiques, ainsi que les mesures considérées efficaces représentent souvent peu ou mal les préoccupations et les besoins des minorités. Il importe donc de s'interroger sur les mécanismes permettant de considérer les voix des divers groupes de la société, dans la crise actuelle et lors de futures pandémies.

Face à ces enjeux émergents, une position plus pro-active que réactive s'impose afin de tenir compte, autant que faire se peut, de toutes les voix dans la société québécoise. Il importe ainsi d'examiner certaines questions pour nourrir les processus décisionnels dans les prochains mois ou lors d'une prochaine pandémie :

- Quels processus d'analyse permettraient de pondérer, d'une part, les risques sur la santé physique, mentale et sociale liés au virus et confinement, et, de l'autre, les bénéfices éventuels de mesure d'ouverture pour mitiger les impacts des diverses mesures socio-sanitaires sur diverses communautés?

- Comment impliquer les groupes minoritaires ou marginalisés dans l'élaboration de mesures de santé publiques qui tiennent compte de leur réalité, et qui soient donc réalistes pour l'ensemble de la population visée?

\section{Vers une approche globale et inclusive en santé publique}

La pandémie a mis en lumière l'importance d'analyser les risques et bénéfices associés à des mesures individuelles et collectives visant le bien-être physique, mental et social des populations. S'il est sûr que les données probantes en santé publique doivent prévaloir, la prise en compte des effets collatéraux du confinement requiert une analyse continue et nuancée d'un phénomène en évolution très rapide, de façon à mitiger ces dommages. Cette analyse doit être transparente et regrouper des acteurs centraux de la vie en société afin d'assurer une légitimité et une adhésion maximale aux mesures adoptées. Outre les risques relatifs de transmission du virus et les impératifs économiques déjà considérés par les décideurs, l'analyse préliminaire des dommages collatéraux du confinement suggère que ces grands principes devraient faire partie de toute analyse de priorité :

- La préservation ou l'amélioration de la santé mentale (diminution de la détresse et des symptômes et amélioration du fonctionnement social, ceux-ci n'étant pas forcément liés);

- La protection de la sécurité des individus mineurs ou vulnérables (en perte d'autonomie à cause de l'âge ou d'un handicap);

- La promotion du soutien au développement des enfants et des jeunes;

- Le soutien à la résilience communautaire;

- La modération des tensions intercommunautaires et la préservation de la cohésion sociale.

Le poids relatif de ces enjeux se modifie avec le temps qui passe. Ainsi, si les problèmes de santé mentale s'imposent d'ores et déjà comme un défi majeur, la question du développement des enfants, des soins et de la solitude des personnes âgées ou du soutien à la résilience communautaire, qui ne se posent pas comme des urgences, doivent cependant être pensé. Par ailleurs, parmi les stratégies favorisant une approche inclusive et une concertation des communautés minoritaires, les pistes suivantes peuvent notamment être envisagées :

- Impliquer et consulter les leaders communautaires et religieux, voir des comités de citoyens, au sujet de la mise en place de mesures touchant les communautés locales, tout en élaborant un mécanisme pour remédier aux différents éventuels concernant les priorités;

- Collaborer avec les tables de concertation des différents quartiers et secteurs, afin de favoriser la communication et la concertation entre les organes de santé publique, les services de santé et services sociaux, les milieux scolaires, les acteurs communautaires et la population;

- Miser sur les CLSC et sur leur mission initiale d'offrir des services locaux, adaptés aux populations desservies, notamment en travaillant avec les agents communautaires et en favorisant les échanges entre services institutionnels et milieux communautaires; 
- Promouvoir, à plus long terme, une meilleure représentation des communautés minoritaires dans les institutions de santé et services sociaux.

De tels mécanismes doivent être révisés afin de s'assurer que les recommandations sanitaires et mesures de soutien sont accessibles et adaptées à la réalité. Dans un contexte de crise fluide, tout processus de consultation doit tenir compte des limites du possible et faire le deuil de l'exhaustivité au profit d'itérations répétées de dialogue, de médiation et de prises de décision au sujet de l'action. Il s'agit non seulement de tenir compte des voix des individus et des communautés, mais aussi de créer des conditions propices à ce que les voix des personnes et groupes plus vulnérables soient entendus par les autorités publiques. Les mécanismes et processus de consultation devraient être réguliers, sans entraver la prise de décision. Afin de nous préparer aux prochaines crises, il est nécessaire d'ores et déjà de développer des liens forts entre institutions publiques et communautés, tant majoritaires que minoritaires, afin de définir les mécanismes favorisant l'émergence d'une santé publique réellement inclusive, qui tienne compte de la santé physique, mentale et sociale de la population.

\section{Références bibliographiques}

Atlani-Duault, L., Mercier, A., Rousseau, C., Guyot, P., \& Moatti, J.-P. (2015). Blood libel rebooted: traditional scapegoats, online media, and the H1N1 epidemic. Culture, Medicine and Psychiatry, 39(1), 43-61.

Azar, K. M., Shen, Z., Romanelli, R. J., Lockhart, S. H., Smits, K., Robinson, S., et al. (2020). Disparities in outcomes among COVID-19 patients in a large health care system in California: study examines disparities in access and outcomes for COVID-19 patients who are members of racial and ethnic minorities and socioeconomically disadvantaged groups. Health Affairs. https://doi.org/10.1377/ hlthaff.2020.00598.

Campbell, H. (2020). The distanced church: reflections on doing church online. Retrieved from: https:/oaktrust.library.tamu.edu/bitstream/ handle/1969.1/187891/Distanced\%20Church-PDF-Magazine\% 20style-FINAL \%20version.pdf?sequence $=5$.

Cleveland J, Hanley J, Jaimes A, Wolofsky T. (2020). Impacts de la crise de la COVID-19 sur les « communautés culturelles » montréalaises Enquête sur les facteurs socioculturels et structurels affectant les groupes vulnérables. In: Sherpa Iu (Ed.), Montreal (Qc).

Cohn, S., \& Kutalek, R. (2016). Historical parallels, Ebola virus disease and cholera: understanding community distrust and social violence with epidemics. PLoS Currents, 8.

Gunnell, D., Appleby, L., Arensman, E., Hawton, K., John, A., Kapur, N., et al. (2020). Suicide risk and prevention during the COVID-19 pandemic. Lancet Psychiatry, 7(6), 468-471.

Institut national d'excellence en santé et en services sociaux. (2020). COVID-19 et les approches favorisant l'observance des mesures de précaution et de protection auprès des personnes en situation de vulnérabilité. Retrieved from https://www.inesss.qc.ca/en/covid-19/ services-sociaux/approches-favorisant-lobservance-des-mesuresde-precaution-et-protection-aupres-des-personnes-en-situation-devulnerabilite.html.

Pfefferbaum, B., \& North, C. S. (2020). Mental health and the Covid-19 pandemic. New England Journal of Medicine, 383, 510-512. https://doi.org/10.1056/NEJMp2008017.

Roy, M., Moreau, N., Rousseau, C., Mercier, A., Wilson, A., \& AtlaniDuault, L. (2020). Ebola and localized blame on social media: analysis of twitter and Facebook conversations during the 2014-2015 Ebola epidemic. Culture, Medicine and Psychiatry, 44(1), 56-79.

World Health Organization. (2020). Practical considerations and recommendations for religious leaders and faith-based communities in the context of COVID-19: interim guidance, 7 April 2020. Retrieved from https://www.who.int/publications/i/item/practicalconsiderations-and-recommendations-for-religious-leadersandfaith-based-communities-in-the-context-of-covid-19.

Publisher's note Springer Nature remains neutral with regard to jurisdictional claims in published maps and institutional affiliations. 